\title{
Relationship between deep medullary veins in susceptibility-weighted imaging and ipsilateral cerebrovascular reactivity of middle cerebral artery in patients with ischemic stroke
}

\author{
XIANJUN HAN, LINHUI OUYANG, CHUNNING ZHANG, HAILING MA and JINGCUI QIN
}

Department of Neurology, The First People's Hospital of Xuzhou, Xuzhou, Jiangsu 221002, P.R. China

Received December 7, 2015; Accepted March 28, 2016

DOI: 10.3892/etm.2016.3198

\begin{abstract}
Deep cerebral veins have been recently associated with the severity of hemodynamic impairment in moyamoya disease. The aim of the current study was to determine the correlation of deep medullary veins (DMVs) in susceptibility-weighted imaging (SWI) with ipsilateral cerebrovascular reactivity (CVR) of and anterior cecebrocervical artery stenosis in patients with ischemic stroke. Patients with unilateral TIA or infarction who underwent $3.0 \mathrm{~T}$ magnetic resonance imaging SWI, digital subtraction angiography and transcranial Doppler with $\mathrm{CO}_{2}$ stimulation within the first 7 days of hospitalization were retrospectively selected. CVR and stenosis of anterior cerebrocervical arteries were compared between different DMVs stages in symptomatic hemispheres (SHs) and asymptomatic hemispheres (AHs). A total of 61 patients were subsequently included in the present study. A univariate analysis was conducted and results for age $\left(\mathrm{P}_{\mathrm{AHs}}=0.004, \mathrm{P}_{\mathrm{SHs}}=0.006\right)$, hypertension $\left(\mathrm{P}_{\mathrm{AHs}}=0.008\right.$, $\left.\mathrm{P}_{\mathrm{SHs}}=0.020\right)$, current smoking $\left(\mathrm{P}_{\mathrm{AHs}}=0.006, \mathrm{P}_{\mathrm{SHs}}=0.021\right), \mathrm{CVR}$ $\left(\mathrm{P}_{\mathrm{AHs}}=0.000, \mathrm{P}_{\mathrm{SHs}}=0.000\right)$, and artery stenosis $\left(\mathrm{P}_{\mathrm{AHs}}=0.000\right.$, $\left.\mathrm{P}_{\mathrm{SHs}}=0.000\right)$ were obtained. The results suggested statistically significant differences between DMVs grades in SHs and AHs. A subsequent multivariate analysis revealed that $\operatorname{CVR}\left(\mathrm{OR}_{\mathrm{AHs}}=0.925,95 \% \mathrm{CI}_{\mathrm{AHs}}: 0.873-0.981 ; \mathrm{OR}_{\mathrm{SHs}}=0.945\right.$, 95\% $\left.\mathrm{CI}_{\mathrm{SH}}: 0.896-0.996\right)$, and artery stenosis $\left(\mathrm{OR}_{\mathrm{AH}}=3.147\right.$, $\left.95 \% \mathrm{CI}_{\mathrm{AH}}: 1.010-9.806 ; \mathrm{OR}_{\mathrm{SH}}=2.882,95 \% \mathrm{CI}_{\mathrm{SHs}}: 1.017-8.166\right)$ were independent risk factors of DMVs. In conclusion, 3.0 T SWI was useful in detecting the DMVs around the lateral ventricle in patients with atherosclerotic ischemic stroke. CVR and stenosis of anterior cerebrocervical arteries were independent risk factors for ipsilateral DMVs in SHs and AHs.
\end{abstract}

Correspondence to: Dr Xianjun Han, Department of Neurology, The First People's Hospital of Xuzhou, 19 Zhongshanbei Road, Xuzhou, Jiangsu 221002, P.R. China

E-mail: drhanxj@tom.com

Key words: deep medullary veins, susceptibility-weighted imaging, cerebrovascular reactivity, artery stenosis, ischemic stroke

\section{Introduction}

Susceptibility-weighted imaging (SWI) has been introduced to the clinical setting in the last decade (1-3). This technique is extremely sensitive for the utilization of paramagnetic material, such as deoxyhemoglobin and hemosiderin $(4,5)$. Combined with its excellent spatial resolution, SWI may be important in detecting the venues system especially in deep regions of the brain $(6,7)$.

Deep medullary veins (DMVs) are an important part of the deep cerebral veins system, originating from the middle part of the cerebral medullary, converging the microvenuals around the centrum ovale and ultimately cascading into the collecting venous trunks (8). Under physiological conditions, diameters of DMVs lumen were $\leq 0.02 \mathrm{~mm}$ and could not be clearly detected. However, a previous study conducted on moyamoya disease (MMD) suggested that in patients with severe hemodynamic impairment, multiple DMVs were observed beside the lateral ventricle by high-resolution magnetic resonance imaging (MRI) (9). To the best of our knowledge, the relationship between DMVs andhemodynamic condition or cerebrocervical artery stenosis in patients with ischemic stroke have yet to be invstigated.

In the current study, cerebrovascular reactivity (CVR) as an index of hemodynamic condition was obtained by transcranial Doppler (TCD) with $\mathrm{CO}_{2}$ stimulation. Additionally, whether DMVs in SWI correlated with the ipsilateral CVR in patients with ischemic stroke was examined.

\section{Patients and methods}

Patients. The clinical data of 61 consecutive patients from the First People's Hospital of Xuzhou (Jiangsu, China) were retrieved. The inclusion criteria for the study were: i) unilateral (MCA) territory ischemic stroke (cerebral infarction and transient ischemic stroke included); ii) atherosclerotic ischemic stroke with assessable digital subtraction angiography (DSA), SWI and TCD CO 2 stimulation results prior to surgical or interventional treatment within the first 7 days of hospitalization. The exclusion criteria were: i) DSA-demonstrated occlusion in the common cerebral, internal cerebral, or middle cerebral arteries (MCAs); ii) previous arteriovenous malformation, 
Table I. Clinical parameters between patients of different DMVs stages in SHs and AHs.

\begin{tabular}{|c|c|c|c|c|c|c|}
\hline \multirow[b]{2}{*}{ Parameters } & \multicolumn{2}{|c|}{ DMVs stages in SHs } & \multirow[b]{2}{*}{ P-value } & \multicolumn{2}{|c|}{ DMV stages in AHs } & \multirow[b]{2}{*}{ P-value } \\
\hline & Stage $1, \mathrm{n}=31$ & Stage $2, n=30$ & & Stage $1, n=41$ & Stage $2, n=20$ & \\
\hline Age, mean \pm SD & $57.0 \pm 13.2$ & $66.4 \pm 11.4$ & $\mathrm{P}=0.004^{\mathrm{a}}$ & $58.5 \pm 13.1$ & $68.1 \pm 10.9$ & $\mathrm{P}=0.006^{\mathrm{a}}$ \\
\hline Male (\%) & $21(67.7)$ & $21(70.0)$ & $\mathrm{P}=0.849$ & $30(73.1)$ & $12(60.0)$ & $\mathrm{P}=0.297$ \\
\hline Hypertension (\%) & $16(51.6)$ & $25(83.3)$ & $\mathrm{P}=0.008^{\mathrm{a}}$ & $22(53.7)$ & $19(95.0)$ & $\mathrm{P}=0.020^{\mathrm{a}}$ \\
\hline Diabetes (\%) & $4(12.9)$ & $7(23.3)$ & $\mathrm{P}=0.335$ & $5(12.2)$ & $6(30.0)$ & $\mathrm{P}=0.153$ \\
\hline Current smoking (\%) & $7(22.6)$ & $17(56.7)$ & $\mathrm{P}=0.006^{\mathrm{a}}$ & $12(29.3)$ & $12(60.0)$ & $\mathrm{P}=0.021^{\mathrm{a}}$ \\
\hline Collateral compensation (\%) & $4(12.9)$ & $6(20.0)$ & $\mathrm{P}=0.454$ & $7(17.1)$ & $3(15.0)$ & $\mathrm{P}=0.837$ \\
\hline $\mathrm{CVR}$, mean $\pm \mathrm{SD}$ & $55.9 \pm 16.6$ & $36.1 \pm 14.0$ & $\mathrm{P}=0.000^{\mathrm{a}}$ & $80.3 \pm 17.8$ & $58.2 \pm 17.0$ & $\mathrm{P}=0.000^{\mathrm{a}}$ \\
\hline Stenosis mild (\%) & $25(80.6)$ & $8(26.7)$ & & $34(82.9)$ & $7(35.0)$ & \\
\hline Moderate (\%) & $4(12.9)$ & $11(36.7)$ & & $6(14.6)$ & $6(30)$ & \\
\hline Severe $(\%)$ & $2(6.5)$ & $11(36.7)$ & $\mathrm{P}=0.000^{\mathrm{a}}$ & $1(2.4)$ & $7(35.0)$ & $\mathrm{P}=0.000^{\mathrm{a}}$ \\
\hline
\end{tabular}

DMVs, deep medullary veins; AHs, asymptomatic hemispheres; SHs symptomatic hemispheres; SD, standard deviation. ${ }^{\text {aStatistical significant }}$ difference $(\mathrm{P}<0.05)$.

venous stroke, brain tumor, or demyelinating diseases, which may influence the outcome of venous imaging; iii) previous thyroid, respiratory, or heart disease, which may influence the mean blood flow velocity detected by TCD; iv) multiple microbleeds located around the lateral ventricles, which may influence the semiquantitative assessment of DMVs; and v) poor transtemporal penetration for ultrasound.

Study protocols were approved by the Institution Review Board of the First People's Hospital of Xuzhou. Hemispheres with new or recent onset of symptoms were defined as symptomatic hemispheres (SHs), the opposite hemispheres were defined as asymptomatic hemispheres (AHs). For each patient, risk factors of cerebrovascular disease such as age, gender, history of hypertension, diabetes mellitus, and current smoking were documented.

Imaging assessment. The patients underwent SWI using a Magnetom Trio whole body 3.0 T MRI scanner (Siemens, Erlangen, Germany). The MRI protocol included axial T1/T2-weighted imaging, axial diffusion-weighted imaging, axial and/or coronal T2-FLAIR imaging, MRA and SWI. SWI images were obtained using one set of parameters (40 mT/m gradient. TE/TR/FA: $25 \mathrm{msec} / 56 \mathrm{msec} / 20^{\circ}$; FOV: $230 \times 115 \times 144 \mathrm{~mm}^{3}$; matrix: 512 × $254 \times 72$; voxel size: $0.45 \times 0.45 \times 2 \mathrm{~mm}^{3}$; and section thickness: $2 \mathrm{~mm}$ ). Imaging of maximum intensity projection (MIP) was also accumulated and reconstituted with commercially available hardware and software. Two experienced MRI-specialized neuroradiologists, blinded to the patients' clinical and hemodynamic information, evaluated the imaging of MIP individually. The number of conspicuous DMVs was classified as: stage 1: $\leq 5$ DMVs and stage 2: $>5$ DMVs. Consensus over inter-observer discrepancies was reached through consultation.

No serious complication occurred during the DSA procedure. The stenosis of excranial internal carotid artery (ICA) was analyzed according to the North American Symptomatic Carotid Endarterectomy Trial Collaborators (10). The Warfarin-Aspirin Symptomatic Intracranial Disease Study was employed to assess the stenosis of intracranial vessels (11).
The most severe segments of tandem stenosis were required for the analysis. The proportion of stenosis $\leq 50 \%$ was documented as mild stenosis, $50 \%<$ stenosis $\leq 70 \%$ as moderate stenosis, and $>70 \%$ was documented as severe stenosis. Collateral compensation between hemispheres was also classified based on DSA results. Positive collateral compensation was defined as ipsilateral ICA territory receiving blood flow from contralateral hemispheres or posterior circulation, whereas isolated hemispheres or giving branches to the contralateral sides were documented as negative collateral compensation.

TCD $\mathrm{CO}_{2}$ stimulation assessment. TCD recordings were made from the two MCAs via the transtemporal route. Recordings were made with a commercially available TCD machine (Companion II; DWL ${ }^{\circledR}$ Sipplingen, Germany) with the probe held in position by an external fixation device. The depth of the probe ranged between 52 and $66 \mathrm{~mm}$, with an average of $56 \mathrm{~mm}$. The probes were fixed when the optimal MCA blood flow was detected. Air was initially administered via a one-way valve mask. Patients breathed through the mask until MCA velocity became stable. A further $30 \mathrm{sec}$ of recording was made at this stage. The mean blood flow velocity was recorded as microvascular flow velocity $\left(\mathrm{MFV}_{1}\right)$. Air/carbon dioxide mixture (5\% carbon dioxide and 95\% oxygen) was then administered via the mask. When the MCA velocity was again stabilized, a further $30 \mathrm{sec}$ of recording was made, and the mean blood flow velocity at that time point was recorded as $\mathrm{MFV}_{2}$. The process was repeated at least three times, and the average value of each parameter was recorded. Patients were required to rest $>5$ min between each process. The CVR value was defined as $\left(\mathrm{MFV}_{2}-\mathrm{MFV}_{1}\right) / \mathrm{MFV}_{1} \mathrm{x} 100$.

\section{Clinical data collection}

Statistical analysis. Data were analyzed using the Statistical Package for the Social Sciences version 18.0 software for windows (SPSS, Inc., Chicago, IL, USA). The statistical agreement of the two observers was analyzed using Cohen's $\kappa$ value. The difference of the clinical and hemodynamic parameters between DMV stages was analyzed using the Student's t-test, 
Table II. Multivariable analysis for risk factors of DMVs in SHs and AHs.

\begin{tabular}{|c|c|c|c|c|c|c|}
\hline \multirow[b]{2}{*}{ Factors } & \multicolumn{2}{|c|}{ DMVs stages in SHs } & \multirow[b]{2}{*}{ P-value } & \multicolumn{2}{|c|}{ DMVs stages in AHs } & \multirow[b]{2}{*}{ P-value } \\
\hline & Odd rates & $95 \%$ CI & & Odd rates & $95 \%$ CI & \\
\hline Age & 1.038 & $0.977-1.102$ & $\mathrm{P}=0.233$ & 1.038 & $0.973-1.107$ & $P=0.263$ \\
\hline Hypertension & 1.414 & $0.275-7.269$ & $\mathrm{P}=0.678$ & 2.576 & $0.568-9.166$ & $\mathrm{P}=0.132$ \\
\hline Current smoking & 4.214 & $0.772-23.019$ & $\mathrm{P}=0.097$ & 1.474 & $0.296-7.344$ & $P=0.636$ \\
\hline CVR & 0.925 & $0.873-0.981$ & $\mathrm{P}=0.009^{\mathrm{a}}$ & 0.945 & $0.896-0.996$ & $\mathrm{P}=0.036^{\mathrm{a}}$ \\
\hline Stenosis & 2.882 & $1.017-8.166$ & $\mathrm{P}=0.046^{\mathrm{a}}$ & 3.147 & $1.010-9.806$ & $\mathrm{P}=0.048^{\mathrm{a}}$ \\
\hline
\end{tabular}

DMVs, deep medullary veins; AHs, asymptomatic hemispheres; SHs symptomatic hemispheres; CVR, cerebrovascular reactivity; CI, confidence interval. ${ }^{a}$ Statistical significant difference $(\mathrm{P}<0.05)$.
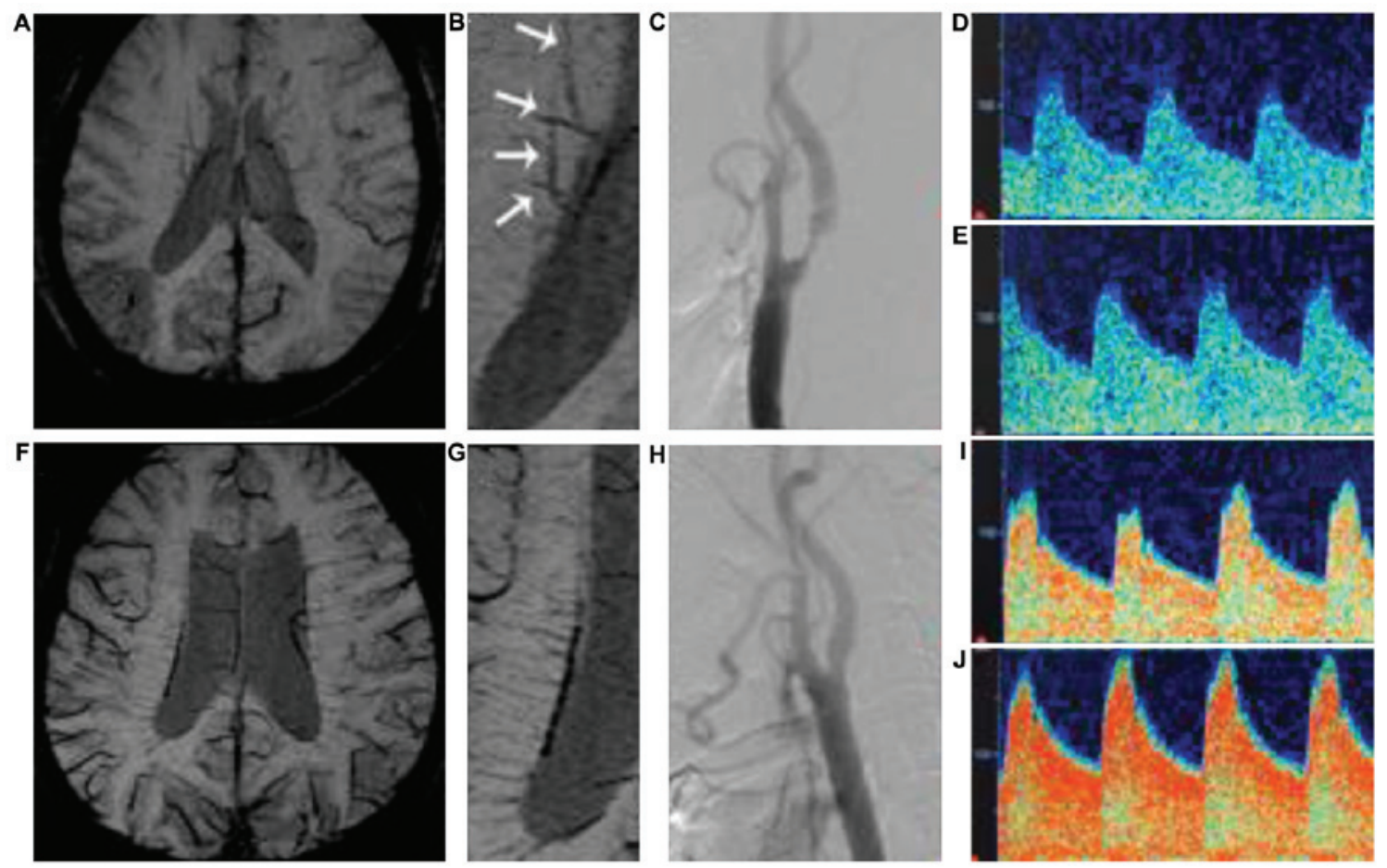

Figure 1. A 72-year-old male was diagnosed with transient ischemic attack of the right hemisphere. Over five deep medullary veins (stage 2) are evident around the (A and B) lateral ventricle in symptomatic hemispheres. Digital subtraction angiography results indicated a severe stenosis at the (C, arrow headed) $\mathrm{C} 1 \mathrm{seg}-$ ment of the internal carotid artery (ICA). (D) Transcranial Doppler (TCD) results before $\mathrm{CO}_{2}$ stimulation showed that $\mathrm{MFV}_{1}=75 \mathrm{~cm} / \mathrm{sec}$. After the stimulation, (E) $\mathrm{MFV}_{2}=87 \mathrm{~cm} / \mathrm{sec}$. Cerebrovascular reactivity (CVR) was calculated to be 16. (F and G) In the asymptomatic hemispheres (AHs), 5 deep medullary veins classified as grade 1 are indicated. Moderate stenosis was detected in the $\mathrm{C} 1$ segment of the (H) ICA. TCD results show that (I) $\mathrm{MFV}=88 \mathrm{~cm} / \mathrm{sec}$ and (J) $\mathrm{MFV}=128 \mathrm{~cm} / \mathrm{sec}$. $\mathrm{CVR}_{\mathrm{AHs}}$ was calculated to be $46 . \mathrm{MFV}$, microvascular flow velocity.

as well as Chi-square and Fisher's exact tests. Logistic regression was employed to determine the independent risk factors for DMVs. For all the analyses, a two-tailed value of $\mathrm{P}<0.05$ was considered to indicate a statistically significant difference.

\section{Results}

A total of 42 males and 19 females were included in the present study. The mean age of the patients was $61.6 \pm 13.1$, with a range of 35-88 years. Based on the DMVs in SHs, 31 patients were classified as grade 1 , and 30 patients as grade 2 . The Cohen $\kappa$ value of this binary classification was 0.806 for SHs, which showed excellent agreement. DSA result suggested that 35 patients had mild stenosis, 15 patients had moderate stenosis, and 14 patients had severe stenosis in the trunk of anterior circulation. CVR was measured to be 46.15 \pm 18.22 . In AHs, 41 patients were classified as DMVs grade 1, and 20 patients as DMVs grade 2 . The Cohen $\kappa$ value was 0.821 . A total of 41 patients with mild stenosis, 12 patients with moderate stenosis, and 8 patients with severe stenosis were detected by DSA. The value of CVR in AHs was 73.03 \pm 20.30 .

The univariate analysis of AHs and SHs revealed that five clinical and imaging indices, including age $\left(\mathrm{P}_{\mathrm{AHs}}=0.004\right.$, $\left.\mathrm{P}_{\mathrm{SHs}}=0.006\right)$, hypertension $\left(\mathrm{P}_{\mathrm{AHs}}=0.008, \mathrm{P}_{\mathrm{SHs}}=0.020\right)$, current smoking $\left(\mathrm{P}_{\mathrm{AHs}}=0.006, \mathrm{P}_{\mathrm{SHs}}=0.021\right), \mathrm{CVR}\left(\mathrm{P}_{\mathrm{AHs}}=0.000\right.$, $\left.\mathrm{P}_{\mathrm{SHs}}=0.000\right)$, and artery stenosis $\left(\mathrm{P}_{\mathrm{AHs}}=0.000, \mathrm{P}_{\mathrm{SHs}}=0.000\right)$ exhibited statistically significant differences between varying DMVs grades (Table I). The subsequent multivariate analysis 
indicated that CVR $\left(\mathrm{OR}_{\mathrm{AHs}}=0.925,95 \% \mathrm{CI}_{\mathrm{AHs}}: 0.873-0.981\right.$; $\left.\mathrm{OR}_{\mathrm{SHs}}=0.945,95 \% \mathrm{CI}_{\mathrm{SHs}}: 0.896-0.996\right)$, and artery stenosis $\left(\mathrm{OR}_{\mathrm{AH}}=3.147,95 \% \mathrm{CI}_{\mathrm{AH}}: 1.010-9.806 ; \mathrm{OR}_{\mathrm{SH}}=2.882,95 \% \mathrm{CI}_{\mathrm{SHs}}:\right.$ 1.017-8.166) were independent risk factors of DMVs (Table II).

\section{Discussion}

The present findings have demonstrated that $3.0 \mathrm{~T}$ SWI was qualified in detecting the DMVs around the lateral ventricle in patients with atherosclerotic ischemic stroke. CVR and stenosis of the trunk of anterior cerebrocervical arteries were independent risk factors for ipsilateral DMVs in SHs and AHs.

In 2010, Kesavadas et al (12), respectively, analyzed the SWI imaging in 18 patients with occlusive/critical stenosis ischemic stroke. Authors of that study initially suggested a significant signal loss and increased diameter of the venous compared with contralateral hemispheres. However, in that study, a comparison of inter-patients was not performed due to the lack of a qualitative or quantitative standard to define a specific segment of veins. In the present study, a semiquantitative classification was applied based on the numerical information of DMVs simplified from Horie's et al (9) results of MMD. The reason for not directly applying previous triple classification (0-5 as stage 1, 6-10 as stage 2 and $>10$ as stage 3$)$ was largely due to the unsatisfied inter-rater reliability. The Cohen $\kappa$ was 0.66 in a previous study (9) and only 0.565 in SHs and 0.569 in AHs in the present study. The findings exhibited unsubstantial agreements, which potentially limit the clinical utility. This abberation may be explained by the limited resolution of 3.0 T MRI especially for obtaining the exact number of small signal loss from 'Brush-like' vessels (Fig. 1A and B). We therefore combined stages 2 and 3 together. Despite the decreased statistical efficiency, the interrater reliability of the binary classification may be deemed reliable.

According to the golden-standard DSA procedure, we concluded that the degree of stenosis was an independent risk factor of DMVs in SHs and AHs. This may be explained by the increased ratio of deoxyhemoglobin/oxyhemoglobin in veins. Previous findings have shown that following severe stenosis of the cerebrocervical arteries, decreased perfusion pressure may upregulate oxygen extraction of the brain tissue (13). A higher concentration of paramagnetic deoxyhemoglobin in venous vessels therefore accounts for the increased visibility of DMVs (9). Our results suggest that in ischemic patients with multiple visible DMVs, attention should be paid to the vascular stenosis of ipsilateral anterior cerebrocevical arteries.

Results obtained by Horie et al (9) showed a negative correlation between DMVs and cerebral reserve assessed by single-photon-emission computed tomography. In the present study, we confirmed CVR as a major part of cerebral reserve, independently contributing to the stages of DMVs, in addition to SHs and AHs. This result supports the specification that conspicuous DMVs indicate venous stasis in that region (9). Decreased CVR induced by hemodynamic impairment shows the over-dilated status of small vessels, which reduces the velocity of the blood flow and ultimately leads to stasis of small veins and therefore severe signal loss in SWI (14). However, TCD was lacking as a method for hemodynamic assessment despite a good correlation of 'gold-standard' positron emission computed tomography (15). Therefore, there may be a discrepancy despite the TCD procedure being performed by the same physician three times. Prospective studies should consider other non-invasive and more accurate methods such as perfusion-weighted imaging of high-resolution MRI.

The other limitation of the present study was that it was performed as a preoperative study with a limited sample size. We were not able to characterize changes of the DMVs, CVR, or stenosis after medical/surgical treatment. Thus, the utility of DMVs in the stratified treatment of ischemic stroke remains unknown. In addition, since CVR and artery stenosis are independent risk factors for subsequent ischemic stroke, whether DMVs stage correlate with worse clinical outcome should also be prospectively confirmed.

\section{References}

1. Abduljalil AM, Schmalbrock P, Novak V and Chakeres DW: Enhanced gray and white matter contrast of phase susceptibility-weighted images in ultra-high-field magnetic resonance imaging. J Magn Reson Imaging 18: 284-290, 2003.

2. Li C, Ai B, Li Y, Qi H and Wu L: Susceptibility-weighted imaging in grading brain astrocytomas. Eur J Radiol 75: e81-e85, 2010.

3. Haacke EM, Mittal S, Wu Z, Neelavalli J and Cheng YC: Susceptibility-weighted imaging: Technical aspects and clinical applications, part 1. AJNR Am J Neuroradiol 30: 19-30, 2009.

4. Ayaz M, Boikov AS, Haacke EM, Kido DK and Kirsch WM: Imaging cerebral microbleeds using susceptibility weighted imaging: One step toward detecting vascular dementia. J Magn Reson Imaging 31: 142-148, 2010.

5. Mori N, Miki Y, Kikuta K, Fushimi Y, Okada T, Urayama S, Sawamoto N, Fukuyama H, Hashimoto N and Togashi K: Microbleeds in moyamoya disease: Susceptibility-weighted imaging versus $\mathrm{T} 2 *$-weighted imaging at 3 Tesla. Invest Radiol 43: 574-579, 2008.

6. Miyasaka T, Taoka T, Nakagawa H, Wada T, Takayama K, Myochin K, Sakamoto M, Ochi T, Akashi T and Kichikawa K: Application of susceptibility weighted imaging (SWI) for evaluation of draining veins of arteriovenous malformation: Utility of magnitude images. Neuroradiology 54: 1221-1227, 2012.

7. Huang P, Chen CH, Lin WC, Lin RT, Khor GT and Liu CK: Clinical applications of susceptibility weighted imaging in patients with major stroke. J Neurol 259: 1426-1432, 2012.

8. Ono M, Rhoton AL Jr, Peace D and Rodriguez RJ: Microsurgical anatomy of the deep venous system of the brain. Neurosurgery 15 : 621-657, 1984.

9. Horie N, Morikawa M, Nozaki A, Hayashi K, Suyama K and Nagata I: 'Brush Sign' on susceptibility-weighted MR imaging indicates the severity of moyamoya disease. AJNR Am J Neuroradiol 32: 1697-1702, 2011.

10. North American Symptomatic Carotid Endarterectomy Trial Collaborators: Beneficial effect of carotid endarterectomy in symptomatic patients with high-grade carotid stenosis. N Engl J Med 325: 445-453, 1991.

11. Schumacher HC, Meyers PM, Higashida RT, Derdeyn CP, Lavine SD, Nesbit GM, Sacks D, Rasmussen P and Wechsler LR: Reporting standards for angioplasty and stent-assisted angioplasty for intracranial atherosclerosis. Stroke 40: e348-e365, 2009.

12. Kesavadas C, Santhosh K and Thomas B: Susceptibility weighted imaging in cerebral hypoperfusion-can we predict increased oxygen extraction fraction? Neuroradiology 52: 1047-1054, 2010.

13. Yamauchi H, Fukuyama H, Fujimoto N, Nabatame H and Kimura J: Significance of low perfusion with increased oxygen extraction fraction in a case of internal carotid artery stenosis. Stroke 23: 431-432, 1992.

14. Kesavadas C, Thomas B, Pendharakar H and Sylaja PN: Susceptibility weighted imaging: Does it give information similar to perfusion weighted imaging in acute stroke? J Neurol 258: 932-934, 2011.

15. Rijbroek A, Boellaard R, Vriens EM, Lammertsma AA and Rauwerda JA: Comparison of transcranial Doppler ultrasonography and positron emission tomography using a three-dimensional template of the middle cerebral artery. Neurol Res 31: 52-59, 2009. 